\title{
El análisis composicional en el estudio de la producción y distribución de la cerámica prehispánica
}

L'analyse de composition chimique dans l'étude de la production et de la distribution de la céramique préhispanique

The role of compositional analysis to study the production and distribution of pre-Hispanic pottery

Iván Ghezzi

\section{(2) OpenEdition}

Journals

Edición electrónica

URL: http://journals.openedition.org/bifea/1565

DOI: 10.4000/bifea.1565

ISSN: 2076-5827

Editor

Institut Français d'Études Andines

Edición impresa

Fecha de publicación: 1 abril 2011

Paginación: 1-29

ISSN: 0303-7495

Referencia electrónica

Iván Ghezzi, «El análisis composicional en el estudio de la producción y distribución de la cerámica prehispánica », Bulletin de l'Institut français d'études andines [En línea], 40 (1) | 2011, Publicado el 01 octubre 2011, consultado el 06 noviembre 2020. URL : http://journals.openedition.org/bifea/ 1565 ; DOI : https://doi.org/10.4000/bifea.1565

\section{cc)}

Les contenus du Bulletin de l'Institut français d'études andines sont mis à disposition selon les termes de la licence Creative Commons Attribution - Pas d'Utilisation Commerciale - Pas de Modification 4.0 International. 


\title{
El análisis composicional en el estudio de la producción y distribución de la cerámica prehispánica
}

\author{
Iván Ghezzi*
}

\section{Resumen}

Actualmente existe un contexto más favorable para la aplicación de técnicas arqueométricas en el Perú, por lo que es oportuna una revisión exhaustiva de su posible contribución al análisis de la cerámica prehispánica. En este artículo se ofrece, en primer lugar, una síntesis de los temas en el análisis de cerámica que pueden estudiarse por métodos arqueométricos. Luego se aborda el análisis composicional como método para definir la procedencia, se enumeran las premisas de este tipo de estudio y se recomiendan los criterios para seleccionar las muestras. A continuación se comparan los métodos mineralógicos y químicos en el estudio de la procedencia. Por último, se describe el análisis estadístico necesario para descubrir los grupos composicionales y la procedencia. Como conclusión, se recomienda identificar el centro de producción usando criterios arqueológicos y geológicos.

Palabras clave: cerámica, arqueometría, análisis composicional, estudio de la procedencia

\section{L'analyse de composition chimique dans l'étude de la production et de la distribution de la céramique préhispanique}

\section{Résumé}

Une conjoncture favorable se prête aujourd'hui au Pérou à l'emploi de techniques relevant de l'archéométrie. Aussi est-il intéressant de faire l'inventaire de ses applications à l'analyse de la poterie préhispanique. Cet article présente tout d'abord une recension des thèmes liés à l'analyse

Pontificia Universidad Católica del Perú, Instituto de Investigaciones Arqueológicas. E-mail: ghezzi@ idarq.org 
de la céramique qui peuvent être étudiés par des méthodes archéométriques. Plusieurs thèmes sont ensuite abordés : I'analyse de la composition chimique afin de déterminer la provenance des pièces ; les prémices de ce genre d'étude ; les critères recommandés pour la sélection des échantillons. Les méthodes minéralogiques et chimiques sont ensuite comparées. Enfin, l'article présente le modèle statistique permettant de mettre à jour les groupes de composition chimique et la provenance des pièces. En conclusion, l'auteur propose d'identifier le centre de production en utilisant des critères mixtes, géologiques et archéologiques.

Mots clés : céramique, archéométrie, analyse compositionnelle, étude de provenance

\title{
The role of compositional analysis to study the production and distribution of pre-Hispanic pottery
}

\begin{abstract}
A more favorable context suggests the growing application of archaeometric methods in Peru. This article reviews their possible contribution to the study of pre-Hispanic pottery. It begins synthesizing the multiple aspects of pottery that can be studied with these methods. Then, it describes compositional analysis in provenance research, while outlining criteria for the selection of samples. It also compares mineralogical vs. chemical methods. To assist in handling compositional data, the article reviews statistical approaches to the identification of ceramic compositional groups and provenience. As a conclusion, it suggests how to identify a production center in light of geological and archaeological criteria.
\end{abstract}

Keywords: pottery, archaeometry, compositional analysis, provenance studies

\section{INTRODUCCIÓN}

El estudio de la cerámica sigue siendo un campo de investigación muy productivo en la arqueología, tanto por su valor clásico como herramienta cronológica e histórico cultural, como por su gran potencial para revelar aspectos fundamentales de la producción y especialización artesanal, las relaciones de intercambio, la organización social, la identidad étnica, la ideología, etc.

Los primeros estudios arqueométricos en este material se remontan a fines del siglo XVIII (Caley, 1967), pero recién a mediados del siglo XX, con el uso de técnicas modernas como la activación neutrónica y la fluorescencia de Rayos $\mathrm{X}$ (Sayre et al., 1957; Young \& Whitmore, 1957; Perlman \& Asaro, 1969) se inició un crecimiento continuo hasta hoy. Este desarrollo se debió fundamentalmente al impacto del positivismo en la Nueva Arqueología de 1960, cuando los arqueólogos empezaron a buscar datos cuantificables en el estudio de la cerámica (Orton et al., 1993). Otro factor que ha influido es el gran potencial de los métodos arqueométricos para abordar múltiples aspectos de la cerámica, como la materia prima, la manufactura, su procedencia y su función. A todo esto se suma actualmente el gran desarrollo de la tecnología que se traduce en una variedad de 
técnicas analíticas, menor costo, multiplicación de laboratorios especializados y la ubicuidad de la computadora, herramienta indispensable en el procesamiento de datos arqueométricos.

En el Perú, los estudios de composición en la cerámica prehispánica se dieron a partir de la década de 1970. Si bien hay trabajos que trataron el tema (Arnold, 1972; Purin, 1985; Salazar et al., 1986; Rozenberg \& Picon, 1990; D'Altroy et al., 1994; Guffroy, 1994; Chapdelaine et al., 2001; Druc, 1998; Druc et al., 2001; Hayashida et al., 2002; Lumbreras et al., 2003; Shimada et al., 2003a; Shimada et al., 2003b; Tschauner \& Wagner, 2003; Wagner et al., 2003; Bray et al., 2005; Vaughn et al., 2005; 2006; Delgado et al., 2007; Rohfritsch, 2006; Thiriet, 2008; Makowski et al., 2008; Pillaca et al., 2008) y hubo un simposio sobre tecnología y organización de la producción de cerámica con considerable enfoque arqueométrico (Shimada, 1994), persiste un notorio retraso respecto a otras latitudes.

Ahora se está gestando un cambio, con la realización de congresos especializados, la creación de una Diplomatura de Arqueometría mediante convenio entre instituciones nacionales y extranjeras, el auge de los estudios ambientales que promueve que los laboratorios adquieran tecnología analítica aplicable a la cerámica, y la realización de proyectos nacionales, a cargo de profesionales como de jóvenes estudiantes (Lumbreras et al., 2003; Montoya et al., 2003; Delgado et al., 2007; Makowski et al., 2008). En medio de un contexto como el descrito, es oportuno divulgar los beneficios de la aplicación de los métodos arqueométricos a temas de investigación cerámica que generalmente son de gran interés entre los arqueólogos en el Perú. Aunque este artículo se centra en el análisis composicional de cerámica, los principios presentados se aplican de manera general al estudio arqueométrico, y por lo tanto al análisis de otros materiales, como metal y obsidiana.

\section{LA CONTRIBUCIÓN ARQUEOMÉTRICA AL ANÁLISIS CERÁMICO}

Antes de enfocarnos en el estudio composicional de cerámica, conviene revisar temas recurrentes en su análisis que los arqueólogos suelen estudiar por métodos convencionales, como la tecnología y organización de la producción, la especialización artesanal, la inferencia de la función y el uso, etc. Estos temas también pueden ser abordados a través de técnicas arqueométricas.

En cuanto a la tecnología de la producción, el proceso de elaboración de una vasija puede ser reconstruido con varias técnicas arqueométricas, para la identificación de las materias primas, de las técnicas de manufactura usadas en la formación, acabado y decoración de vasijas y de la cocción. El primer paso del alfarero es la obtención de materias primas. En general, su mayor preocupación en la selección y procesamiento de arcillas es obtener las propiedades físicas apropiadas, como un balance adecuado entre la plasticidad y contracción de la pasta (Tite et al., 2001). Por lo tanto, para entender la tecnología cerámica es necesario identificar la materia prima y sus propiedades. En cuanto a la arcilla, la cocción transforma totalmente los minerales originales, de manera que es difícil 
reconocerlos (Tite et al., 2001). Sin embargo, se pueden identificar las arcillas ricas en caolinita, que mantienen después de la cocción un contenido alto en aluminio y bajo en alcalinos (Rice, 1987). Estos elementos químicos se pueden reconocer mediante la fluorescencia de Rayos X, activación neutrónica, etc. (García-Heras et al., 2001).

Para alcanzar las propiedades físicas apropiadas, el alfarero a menudo agrega inclusiones a la pasta (minerales, fragmentos de roca, otros), también llamadas antiplásticos y/o temperante (Bishop, 1980). Estas se pueden identificar y cuantificar con un análisis petrográfico (Riederer, 2004). Generalmente, las partículas en el rango 0.02-0.06 mm son consideradas como parte de la matriz de arcilla, y las mayores a $0.06 \mathrm{~mm}$ como inclusiones. Es indispensable, para el interesado en el proceso productivo, saber si las inclusiones son agregadas por el alfarero, o son intrínsecas a la arcilla empleada en la preparación de la pasta. Es posible medir con microscopio óptico y microscopio electrónico de barrido el tamaño y la morfología de las partículas mayores, y así poder distinguir entre las inclusiones naturales y agregadas (Rye, 1981; Rice, 1987; Froh, 2004; Riederer, 2004). Sin embargo, la identificación del material agregado se puede complicar cuando se trata de materiales similares entre sí. Uno de estos casos es cuando la arcilla y el temperante mineral tienen un mismo origen intrusivo.

Preparada la pasta, el alfarero construye la vasija a partir de la misma. El método usado en la formación puede identificarse por la orientación de los poros e inclusiones de la pasta (Rye, 1981; Rice, 1987). El anillado produce una alineación horizontal detectable mediante el microscopio de luz polarizada, el microscopio electrónico de barrido, etc. (Tite, 1999). Otro método es la radiografía, que revela la técnica de manufactura, las uniones entre las distintas secciones usadas en la formación de la cerámica, y la manera en que se adjunta asas, picos, y otros apéndices (Carr, 1990; Deschler-Erb et al., 2004; Middleton, 2005). Así, con la ayuda de radiografías de vasijas enteras de Tablada de Lurín, Makowski identificó el uso del paleteado en la manufactura (Makowski, 2002).

Para darle acabado a la superficie de la vasija antes de la cocción, el alfarero recurre a una serie de técnicas. Estas pueden revelarse en una sección delgada con un microscopio electrónico de barrido. Por ejemplo, el bruñido y el pulido generan un brillo característico, resultado de la compresión y alineación de las partículas superficiales de arcilla, lo que puede identificarse en el microscopio (Rye, 1981; Rice, 1987). La aplicación de engobe, por otro lado, se puede distinguir bajo el microscopio porque su estructura es más fina y densa que la pasta. Más aún, su composición química es a menudo diferente, lo que puede detectarse con métodos como la espectrometría de masas (Speakman \& Neff, 2005b). Los pigmentos minerales aplicados, sean parte del engobe o la decoración, pueden identificarse por microscopia electrónica de barrido (Froh, 2004), espectroscopía Raman (Smith \& Clark, 2004; Weerd et al., 2004), espectrometría de masas (Vaughn et al., 2005) y fluorescencia de Rayos-X (García-Heras et al., 2001). En muestras de cerámica Moche de la huaca de la Luna, Chapdelaine identificó con un microscopio electrónico de barrido el uso de calcita y óxido de hierro para producir pigmentos blancos y rojos (Chapdelaine et al., 1997). 
El paso final en la elaboración de la vasija es su cocción, para la cual el alfarero tiene a su disposición una serie de opciones que generan condiciones particulares al quemar la pieza. Para reconstruir las condiciones de cocción, es necesario establecer la relación entre la temperatura y los cambios mineralógicos y microestructurales. La difracción de Rayos X (Stanjek \& Häusler, 2004) y la espectroscopía Mössbauer (Maggetti, 1982; Wagner et al., 2000; Wagner \& Kyek, 2004) pueden identificar cambios mineralógicos, mientras que los microestructurales pueden detectarse con el microscopio electrónico de barrido (Tite, 1982; Froh, 2004). Shimada usó espectroscopía Mössbauer para comparar el estado físico y químico de i) especímenes producidos en experimentos de cocción de cerámica en el campo; ii) especímenes producidos en el laboratorio, bajo condiciones controladas, y iii) especímenes de los periodos Formativo y Sicán Medio en Batán Grande (Shimada et al., 2003a).

La atmósfera de cocción se determina convencionalmente por el color de la pasta, aunque la combinación de colores es difícil de interpretar. La espectroscopía Mössbauer, que brinda información sobre el estado de oxidación del hierro presente, distingue, por ejemplo, entre una cocción oxidante y una cocción reductora seguida de atmósfera oxidante en el enfriamiento (Wagner \& Kyek, 2004). En muestras de cerámica de Huaca Loro en Batán Grande, Shimada identificó por espectroscopía Mössbauer que la mayoría de las vasijas fueron cocidas en atmósfera reductora, mientras que las usadas solo como ofrenda funeraria, probablemente hechas en serie y de prisa, fueron apenas secadas a una temperatura por debajo de $400^{\circ} \mathrm{C}$ (Shimada et al., 2003b). Por otro lado, Tschauner encontró en un taller de cerámica chimú en Lambayeque que se usaba predominantemente una atmósfera reductora, con una reoxidación parcial al final del ciclo de cocción, lo que permitió identificar con precisión el tipo de horno compatible con estos resultados (Tschauner \& Wagner, 2003).

La organización de la producción de cerámica es un tema que genera mucho interés entre los arqueólogos, por la supuesta relación entre el grado de especialización artesanal y las formas de organización económica, social y política (Leeuw \& Pritchard, 1984; Pool, 1992; Arnold, 2000; Eerkens \& Bettinger, 2001; Roux, 2003; Underhill, 2003). Varios autores han clasificado el modo de producción de la cerámica basados en distintos parámetros, como el tamaño de la unidad de trabajo, el contexto social de los productores, etc. (Peacock, 1982; Rice, 1991; Costin, 2001). Algunos parámetros, como el grado de estandarización, la concentración o dispersión de los centros de producción, y el radio de distribución de la cerámica, pueden ser evaluados por métodos arqueométricos. En el Alto Mantaro, D'Altroy y Bishop pudieron demostrar gracias a la activación neutrónica que la cerámica Inca era producida con materias primas distintas a las usadas en la cerámica local. Esto indica que los incas controlaban a nivel regional, empezando por el control de las fuentes de materias primas, la producción de su cerámica emblemática, y que esta se distribuía selectivamente (D'Altroy \& Bishop, 1990). Por el contrario, usando métodos convencionales y análisis composicional con LAICP-MS, Makowski identifica un escenario distinto en la costa central durante la 
dominación inca, puesto que halla gran variabilidad tecnológica y estilística en la cerámica del periodo, lo que permite descartar una producción centralizada por el poder político (Makowski et al., en prensa).

La inferencia de la función es un aspecto importante, pero elusivo, en la interpretación de la cerámica y su contexto social (Lumbreras, 2005). Requiere la consideración cuidadosa del contexto, forma, dimensiones y propiedades físicas y mecánicas de las vasijas y residuos de los contenidos originales (Henrickson \& McDonald, 1983; Hally, 1986; Beaudry, 1988; Mills, 1989; Neupert, 1994; Rice, 1996; Saunders \& Hays, 2004). La composición, tamaño y abundancia de las inclusiones en la pasta tiene un efecto directo sobre algunos indicadores de la función, como la resistencia a la temperatura y al estrés térmico, la porosidad y la densidad de las vasijas (Rye, 1981; Rice, 1987). El análisis composicional identifica las propiedades físicas asociadas a categorías funcionales de vasijas, especialmente cuando los grupos composicionales se correlacionan con la forma, el contexto y datos etnoarqueológicos (Polvorinos del Río et al., 2005). Por otro lado, las propiedades mecánicas de las vasijas son también indicadores importantes que permiten inferir su función, o al menos excluir funciones que son incompatibles (Tite et al., 2001; Kilikoglou \& Vekinis, 2002).

Numerosos estudios experimentales y etnoarqueológicos muestran que las condiciones de uso alteran la cerámica en forma característica (Hally, 1983; Skibo, 1992; Crown \& Bishop, 1994; Kobayashi, 1994; Beck et al., 2002). Así, los estudios de alteración sirven para inferir de modo general la función y uso de las vasijas en un contexto sistémico de consumo (Schiffer, 1989). Aunque generalmente se presta más atención a las alteraciones de la superficie exterior, como el desgaste, el hollín, las rajaduras, etc., los residuos orgánicos depositados al interior de las vasijas por el contenido, sea alimenticio u otro, pueden examinarse mediante técnicas arqueométricas (Skibo, 1992; Heron \& Evershed, 1993; Evershed et al., 1995; Brown \& Heron, 2003; Roumpou et al., 2003). Por ejemplo, los lípidos preservados en las vasijas por el contacto con grasas, aceites, ceras y resinas de productos orgánicos pueden ser identificados mediante la cromatografía de gases y la espectrometría de masas (Charters et al., 1997; Dudd et al., 1999; Evershed et al., 2000). Cuando la separación de los lípidos es seguida de un análisis de isótopos estables de carbono, es posible identificar residuos de maíz (Reber et al., 2004). Si la cerámica ha sido empleada en la preparación, almacenamiento o consumo de bebidas, como la chicha, su presencia se puede identificar en los residuos mediante un análisis de polen, de levaduras y pruebas de fermentación, como hizo Segura con cerámica de Cajamarquilla (Segura, 2001). Los fitolitos y otros biosilicatos preservados en el residuo orgánico de las vasijas pueden permitir identificar maíz, calabaza, mate, palma, etc. mediante el microscopio óptico y el microscopio electrónico de barrido (Staller \& Thompson, 2002; Bozarth \& Guderjan, 2004). Finalmente, el análisis químico de los residuos orgánicos es también prometedor (Cackette et al., 1987; Regert et al., 2003), aunque ha sido considerado incierto para elementos como el fósforo (Dunnell \& Hunt, 1990). 


\section{PRINCIPIOS BÁSICOS EN EL ESTUDIO DE LA PROCEDENCIA}

Como demuestra la síntesis previa, la arqueometría permite abordar una variedad de temas cerámicos. El área de estudio que genera mayor interés entre los arqueólogos es la reconstrucción de las redes de producción y distribución de cerámica a través de la identificación del lugar de su manufactura o «procedencia». Esto permite al arqueólogo vincular los centros de producción con los asentamientos en que se utiliza la cerámica, y por lo tanto ofrece datos valiosos sobre la interacción social y económica entre comunidades.

Los centros de producción se identifican a través de un análisis composicional, definiendo conjuntos de cerámica compuesta por una misma combinación básica de arcilla y temperante, mediante un análisis químico de las concentraciones de elementos, o un análisis mineralógico y cuantitativo de las inclusiones. Cada conjunto es un grupo composicional, originado en un mismo centro de producción.

Obviamente, la relación entre los grupos composicionales y los lugares de procedencia no es tan directa, ni está libre de complicaciones, pues hay varias premisas involucradas. Para que la identificación de los centros de producción sea exitosa, se deben cumplir algunas condiciones en cuanto a la disponibilidad de las fuentes de materia prima, la forma de preparación de la pasta, la estabilidad durante la cocción, etc. Estas condiciones han sido enumeradas recientemente. La condición más importante es el llamado «postulado de procedencia», según el cual, cuando la historia geológica de las fuentes de arcilla es suficientemente diversa, la diferencia entre fuentes excede la variación al interior de cada una, permitiendo asignar grupos de cerámica a una fuente en particular mediante el análisis composicional (Weigand et al., 1977; Bishop et al., 1982). En la práctica, en algunos casos la composición puede variar en diferentes vetas de la misma fuente de arcilla, de manera que la confiabilidad de los métodos arqueométricos para distinguir entre depósitos de arcilla también puede variar. Cuando esto ocurre, no significa que la determinación de la procedencia sea imposible, pues el problema se puede resolver durante el análisis estadístico de los datos y con la elaboración sistemática de colecciones de referencia. En la costa peruana generalmente se asume que la mineralogía es esencialmente la misma de un valle a otro. Sin embargo, estudios recientes realizados en los valles de Moche, Santa y Lurín son prometedores en la diferenciación de fuentes de arcilla entre valles vecinos e incluso del mismo valle (Makowski et al., 2008; Chapdelaine et al., 2009). Estudios preliminares como éstos deben ser complementados con exploraciones, muestreos y pruebas de arcillas en el área de estudio - como los trabajos de Makowski en la costa central, Vaughn en Nazca y Rohfritsch en el valle de Jequetepeque- para que el resultado pueda ser aceptado plenamente (Vaughn \& Neff, 2004; Rohfritsch, 2007; Makowski et al., 2008).

Otra condición fundamental es que la composición de la pasta no varíe por efecto de la cocción o las condiciones posdeposicionales. En cuanto a la estructura y composición química, la variación es inevitable, pues las altas temperaturas producen 
cambios mineralógicos y microestructurales en la matriz de arcilla de la pasta. Aún así, los experimentos indican que la mayoría de elementos químicos no se volatilizan significativamente durante la cocción, con la posible excepción de arsénico, azufre, bromo y cloro, cuyo punto de ebullición es relativamente bajo (Rye \& Duerden, 1982; Kilikoglou et al., 1988; Storey, 1988; Cogswell et al., 1996; Schwedt et al., 2004). Los cambios ocasionados por el impacto térmico generalmente no afectan a la mayoría de las inclusiones minerales en la pasta, por lo que su identificación por el microscopio petrográfico resulta confiable.

Otros estudios han detectado alteraciones posdeposicionales, o diagénesis, en la cerámica. En la costa peruana, por ejemplo, es común la salinización. En general, la diagénesis ocurre muy raramente, y solo afecta algunos elementos químicos, como fósforo, bario, calcio, cesio, potasio, rubidio y sodio (Lemoine \& Picon, 1982; Franklin \& Vitali, 1985; Picon, 1987; Kilikoglou et al., 1988; Buxeda i Garrigós, 1999; Schwedt et al., 2004). Por lo tanto, el comportamiento estadístico de estos elementos debe vigilarse con cuidado en la exploración estadística inicial de los datos, para decidir si deben incluirse o no en el análisis.

Una condición de tipo cuantitativo, si se trata de un análisis químico, consiste en medir simultáneamente 20 a más elementos, con la instrumentación más precisa posible (Harbottle, 1991). Esto asegura la calidad necesaria en los datos para definir grupos composicionales. En la práctica, algunas técnicas arqueométricas no pueden distinguir tantos elementos con la necesaria precisión, pero otras, como la activación neutrónica y la espectroscopia de masas, pueden distinguir con precisión más de 30 elementos químicos. En el análisis mineralógico se puede dar el caso que una inclusión sea diagnóstico de una fuente, pero generalmente es preferible hacer una descripción petrográfica completa de todas las inclusiones en la pasta (Mommsen, 2001).

Una premisa importante en la determinación de la procedencia es que las materias primas son locales y no se comercializan a largas distancias. A juzgar por los resultados de estudios etnoarqueológicos, esta premisa se aplica en la mayoría de casos. Arnold encontró en Ayacucho que las fuentes de arcilla se encontraban en un radio de $1 \mathrm{~km}$ en $33 \%$ de los casos, y de $7 \mathrm{~km}$ en 84 \% (Arnold, 1993). Asimismo, el $97 \%$ de los temperantes se obtenía en un radio de 9 km. Aún así, Shimada advierte que no debe descartarse el caso de alfareros itinerantes, que transportan sus arcillas preferidas de un sitio a otro o las dejan en varios lugares a lo largo de sus rutas (Shimada et al., 1994). Asimismo, Druc ha reportado el intercambio de materias primas entre los centros de producción en Huari, Ancash (Druc, 2001).

Otra premisa se refiere a la variabilidad en la combinación de arcilla e inclusiones con que se prepara la pasta. Hankey ha indicado que generalmente se sigue una receta específica, determinada por experimentación, en la selección de las materias primas (Hankey, 1983). Mommsen, a partir de la revisión de una gran base de datos composicionales en la Universidad de Bonn, considera que la premisa sobre una pasta homogénea para cada serie de producción es justificada (Mommsen, 2001; 2004). En algunas regiones del mundo no se podrá definir 
patrones composicionales con facilidad, pues se producen superposiciones de concentración entre elementos, pero en general una serie discreta de producción produce grupos composicionales definidos, que se pueden identificar mediante análisis arqueométricos. Al ser las «recetas» parte de una tradición tecnológica (Lechtman, 1979; Gosselain, 1992; Lemonnier, 1993), la composición básica de la pasta tiende a perdurar en el tiempo.

La enumeración y discusión de estas condiciones generales y premisas no significa que la identificación de la procedencia de grupos composicionales está libre de incertidumbre, pues los alfareros no siguen al pie de la letra las reglas en la preparación de la pasta. Por ejemplo, la abundancia de inclusiones en las pastas varía dentro de un mismo taller. Este problema, conocido como dilución, afecta al análisis composicional. Existen técnicas estadísticas para corregir el problema (Sayre, 1988; Mommsen et al., 1991; Beier \& Mommsen, 1994; Buxeda i Garrigós, 1999), pero la solución idónea es el uso de instrumentos que permita diferenciar entre la matriz de arcilla y las inclusiones, como la microsonda electrónica, la espectroscopia de masas, etc. (Freestone \& Middleton, 1987; Speakman \& Neff, 2005b).

En otros casos, el alfarero introduce variabilidad en la pasta al usar diferentes mezclas de arcillas. Los cambios pueden deberse al deseo de producir cerámica con propiedades variadas, de acuerdo a criterios tecnológicos, culturales y de función (Tite et al., 2001). En este caso, el análisis podría identificar varios grupos composicionales procedentes de un mismo centro de producción. Este problema se puede detectar en el análisis estadístico, al examinar la dispersión de los grupos composicionales: en una pasta homogénea, ésta no supera generalmente el $10 \%$. Una dispersión mayor sugiere la coexistencia de varios grupos en un mismo material, sean estos de una misma zona de producción o varias (Mommsen, 2001; 2004).

En síntesis, factores tecnológicos y culturales, como el transporte y la mezcla de arcillas o la adición de inclusiones, complican la relación aparentemente directa entre las fuentes de arcilla, los talleres y los grupos composicionales. Para la mayoría de los casos, sea que los alfareros utilicen una sola fuente, o mezclen varias arcillas, incluso de fuentes geológicamente distintas, el resultado de la preparación es una pasta relativamente homogénea que puede ser definida por análisis composicional. En algunos casos puntuales, sobre todo si se prepara una pasta distinta para cada clase de vasija, el análisis composicional puede detectar varios grupos por cada centro de producción. Afortunadamente, estos casos pueden ser identificados y tratados apropiadamente, examinando la dispersión de los grupos composicionales.

\section{ALGUNOS CRITERIOS PARA LA SELECCIÓN DE MUESTRAS}

La selección de piezas relevantes al estudio depende fundamentalmente de la investigación arqueológica en que se inserta el análisis: la muestra será plural si el objetivo es reconstruir las relaciones de intercambio o si es determinar el nivel de especialización artesanal en la sociedad. Sin embargo, hay criterios generales para 
una selección adecuada que se discute a continuación (Bishop et al., 1982; Rice, 1987; Young \& Pollard, 2000).

El muestreo se debe basar en la tipología cerámica elaborada por el arqueólogo a partir del análisis macroscópico de la pasta, manufactura, forma y decoración. En primera instancia, el análisis composicional permite evaluar la variabilidad interna en cada tipo cerámico y afinar la tipología. Por ejemplo, Makowski ha demostrado que el análisis composicional realizado en las muestras de cerámica del valle de Lurín respalda ampliamente los taxones establecidos a partir de un estudio de pastas convencional previo (Makowski et al., 2008). En la figura 1 se observa que las muestras de una pasta se asocian con un grupo composicional y tienen una composición homogénea, ilustrada por el agrupamiento entre las mismas.

Por lo tanto, no se debe elegir las piezas al azar, ni por unos cuantos atributos de la pasta, sino a partir de una tipología fundamentada (Adams \& Adams, 1991). Se requiere varias muestras por cada tipo, de manera que las diferencias resultantes sean válidas estadísticamente. Asimismo, se prefiere especímenes representativos, pero también, por comparación, algunos de los menos típicos o considerados exóticos.

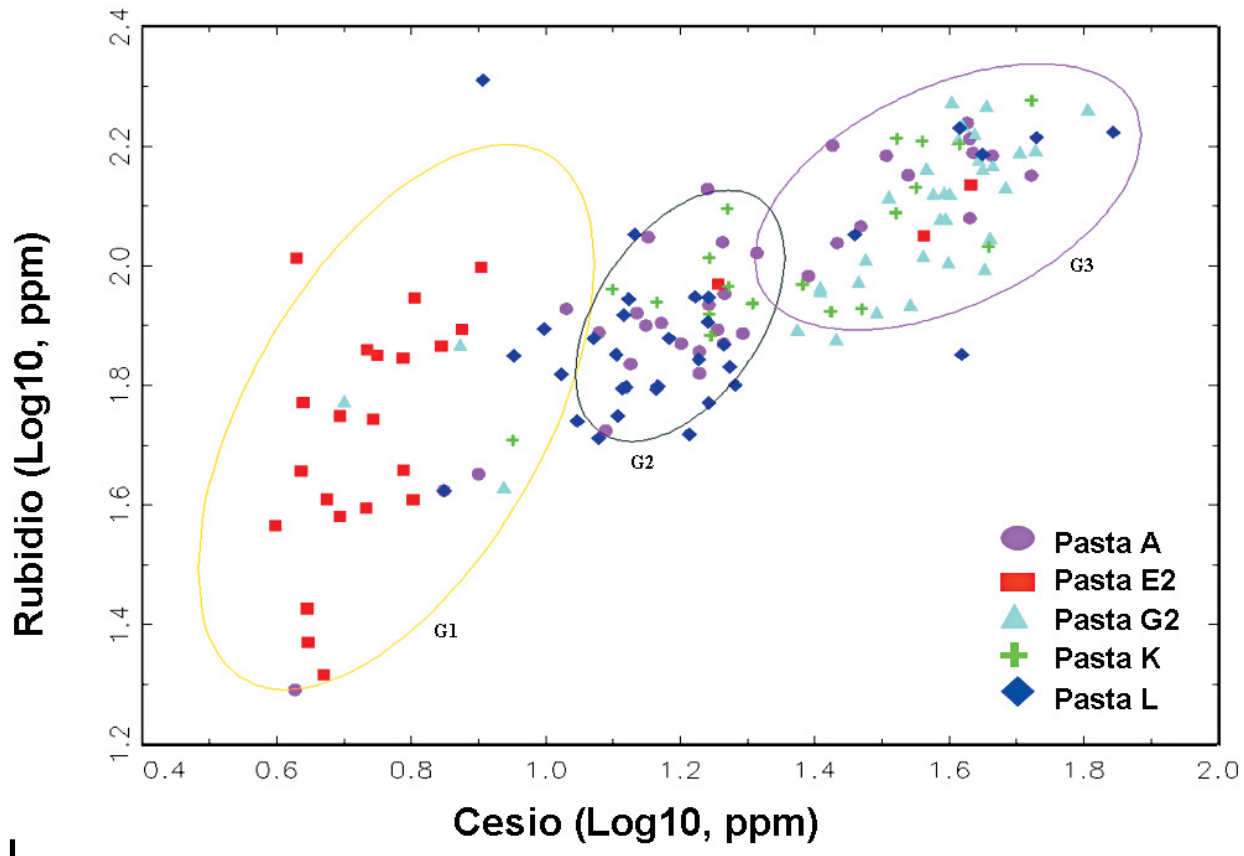

Figura 1 - Diagrama de dispersión de concentraciones de cesio y rubidio en los tres principales grupos composicionales

Las muestras individuales han sido simbolizadas de acuerdo a la pasta definida por métodos convencionales. Las elipses representan un nivel de confianza de $90 \%$ de pertenencia. Los datos composicionales, en partes por millón (ppm), han sido transformados a logaritmos de base 10 (Makowski et al., 2008). 
No puede saberse a priori cuántas muestras son necesarias, pues ello depende de la variabilidad al interior de los tipos y entre tipos, pero se considera que pueden ser necesarias 15-20 muestras por cada uno (Bishop et al., 1982; Tite, 1999). De existir una limitación en el número de muestras a analizar, es preferible evaluar intensivamente algunos tipos directamente relacionados con la problemática de investigación, antes que analizar muy pocos fragmentos de cada tipo.

El análisis composicional también sirve para reconstruir redes de intercambio de cerámica en una determinada región. En este caso, se requiere muestras de varios sitios dispersos por toda la región, tanto de centros de producción y distribución de cerámica como de lugares de consumo. Para una adecuada representación, se requieren de 10 a 15 muestras de cada sitio (Bishop et al., 1982). Idealmente, también se debe analizar muestras de fuentes de arcilla cercanas a cada sitio, aún cuando se considera que su relación con la composición de cerámica no es directa (Mommsen, 2001; 2004).

Sería erróneo excluir de la muestra el material de contexto, diagnóstico o bien conservado, solo porque el análisis arqueométrico es a menudo destructivo. Por el contrario, la interpretación es más sólida cuando se cruzan los datos composicionales con la mayor cantidad posible de datos arqueológicos. Así, se debe seleccionar piezas con atributos diagnósticos de pasta, manufactura, forma, función, etc. Además, deben tener superficies bien preservadas, sin huellas de erosión, incrustación, o mineralización, aunque la mayoría de laboratorios removerá la parte superficial para evitar problemas analíticos. Es preferible usar fragmentos grandes, que reflejan la heterogeneidad composicional que presentan las vasijas. Además, de ellos se pueden obtener muestras para varias pruebas distintas y hasta para duplicar un análisis. Por otro lado, siempre se debe tener en cuenta que las propiedades físicas de la cerámica y los procesos de formación conducen a patrones muy variados de fractura y tamaño final de los fragmentos (Orton, 1993; Schiffer, 1996). Así, cuando un tipo particular de cerámica está representado por fragmentos muy pequeños, se deben tomar precauciones para no excluirlos.

El contexto de la muestra también debe considerarse, por sus connotaciones cronológicas, sociales, funcionales, etc. Las mejores muestras proceden de contextos confiables, como un nivel sellado si procede de excavación, o un sitio monocomponente si es material de superficie. En un estudio reciente de cerámica de Chavín de Huántar, las piezas fueron seleccionadas de acuerdo a su contexto de hallazgo (Galería de las Ofrendas, Pirámide Tello, pueblo de Chavín) y estilo (Wacheqsa, Puksha o Floral), con el fin de evaluar la hipótesis arqueológica sobre el origen foráneo del material Ofrendas (Lumbreras et al., 2003). Tener en cuenta estos criterios permite cruzar los resultados composicionales con atributos cerámicos y finalmente, con un contexto social. En síntesis, el fragmento ideal está bien conservado, tiene una composición y textura de la pasta, técnica de manufactura, y condiciones de cocción características, es diagnóstico de forma, está decorado con un estilo reconocible, y procede de un contexto arqueológico confiable. 


\section{COMPOSICIÓN MINERAL Y COMPOSICIÓN QUÍMICA}

De acuerdo al tipo de información almacenada en la pasta, se distinguen dos métodos usados en el análisis composicional: análisis mineralógico y análisis químico de las concentraciones de elementos (Wilson, 1978). En esta sección se ofrece una comparación entre ambos, pese a que a menudo la selección de un método particular puede no depender de sus ventajas y desventajas, si no de su disponibilidad y/o costo.

La técnica más importante usada en el análisis mineralógico es la petrografía de secciones delgadas (Stoltman et al., 1992; Riederer, 2004). Esta técnica identifica y cuantifica las inclusiones minerales y de fragmentos de roca presentes en la pasta, y permite distinguir, gracias a su tamaño y morfología, entre las inclusiones intrínsecas a la arcilla y las agregadas por el alfarero (Rye, 1981; Rice, 1987; Froh, 2004). Las inclusiones reflejan la geología de la región de la que proceden la arcilla y el temperante, por lo que tiene una incidencia fundamental en la determinación de la procedencia.

La petrografía es generalmente más efectiva con pastas de textura gruesa, que contienen abundantes inclusiones. Aunque en contados casos un tipo de inclusión determinado es tan característico que permite la identificación precisa de la fuente, generalmente es necesaria una descripción petrográfica completa de las inclusiones. Aún así, cuando la región en que se produce la cerámica es geológicamente homogénea, resulta difícil identificar las fuentes mediante petrografía. Del mismo modo, cuando se usa arena, concha, calcita y otros materiales abundantes en la naturaleza como temperante, resulta difícil identificar su origen (Tite, 1999). En el caso de pastas de textura fina, el análisis petrográfico encuentra sus mayores obstáculos. Para obtener una pasta fina, los alfareros seleccionan cuidadosamente la arcilla y la procesan, removiendo inclusiones no deseadas, como fragmentos de roca, que por lo general constituyen la mejor evidencia petrográfica para determinar la procedencia (Rye, 1981; Rice, 1987). Así, las pastas finas por lo general no poseen suficiente variabilidad petrográfica para establecer por este método la procedencia.

Una desventaja adicional de la petrografía es que la interpretación de los datos depende en gran medida de la experiencia, relativamente subjetiva, del especialista en el reconocimiento de las inclusiones minerales. Por el contrario, el análisis químico de elementos (mayores, menores y traza) produce datos cuantitativos con alta compatibilidad entre distintas técnicas y laboratorios (Kennett et al., 2002; Glascock et al., 2004; Speakman \& Neff, 2005a). La mayoría de técnicas químicas permite procesar un gran número de muestras en corto tiempo $y$, a diferencia de la petrografía, el resultado depende más de las características del instrumento empleado que de la experiencia del operador. Además, los métodos de análisis químico tienden a mejorar, automatizarse y reducir costos constantemente. Esto no quiere decir que los métodos químicos están libres de subjetividad, pues el análisis estadístico y la interpretación de los resultados también están sujetos a las elecciones particulares del investigador. 
Muchos investigadores consideran que tanto el análisis mineralógico como el químico son indispensables, y que deben realizarse en simultáneo (Bishop et al., 1982). Se ha propuesto el término «enfoque integral» para los estudios que incluyen ambas categorías de análisis como fases consecutivas y complementarias de una investigación (Tite, 1999). Sin embargo, para algunos defensores de la petrografía, el análisis químico, al ignorar las fuentes minerales de los elementos presentes, no distingue si estos se deben a la arcilla o a las inclusiones (Stoltman \& Mainfort, 2002). Así, según Stoltman, el análisis petrográfico puede hasta contradecir y corregir los resultados del análisis químico de elementos. No sorprende entonces que exista un debate álgido entre los proponentes del análisis químico y el mineralógico en Norteamérica (Neff et al., 2006a; Neff et al., 2006b; Sharer et al., 2006).

Es importante considerar frente a estas alternativas que, mientras los análisis mineralógicos se centran en las inclusiones de la pasta, los métodos químicos más comunes analizan la pasta en su conjunto, tanto el componente de arcilla como las inclusiones. Algunos métodos, como LA-ICP-MS, permiten analizar la matriz de arcilla e inclusiones por separado, además de identificar las inclusiones minerales usando luz polarizada, cual microscopio petrográfico (Speakman \& Neff, 2005b). La habilidad de concentrarse en la arcilla exclusivamente es una ventaja, puesto que la frecuencia de inclusiones en pastas de un mismo taller puede variar por el problema de dilución. Así, la composición química de la cerámica, y por lo tanto su procedencia, dependerá en primer lugar de la arcilla o combinación de arcillas usadas, y en segundo lugar de las inclusiones.

Cuando se logra medir suficientes elementos mediante los métodos químicos, el patrón composicional resultante es tan característico como una huella digital y probablemente, único en el mundo (Mommsen, 2001). Esta huella digital química eventualmente se puede asignar a un lugar de producción y determinar la procedencia. Por esta razón, existe un consenso creciente en Norteamérica y Europa sobre el análisis químico como método para estudiar la procedencia, mientras que el análisis mineralógico es complementario como parte del enfoque integral, y sirve para subdividir los grupos definidos por composición química, o para comprender mejor las razones, a veces ignoradas, de la separación química detectada entre las muestras (Glascock et al., 2004; Mommsen, 2004). Por el contrario, cuando el estudio se centra en la tecnología de producción de cerámica, más que en su procedencia, el análisis mineralógico es insuperable.

\section{MÉTODOS QUÍMICOS EN EL ESTUDIO DE LA PROCEDENCIA}

El análisis composicional se usa, como primer paso, para identificar los diferentes grupos de cerámica en un sitio o una región, afinando las tipologías locales mediante una evaluación de la variabilidad interna en cada tipo cerámico. Como segundo paso, sirve para localizar el centro de manufactura de los grupos de cerámica.

La evaluación composicional de la tipología cerámica de una región puede dar resultados muy valiosos antes de enfocarse en la procedencia. Makowski ha 
explorado la relación entre estilo cerámico e identidad étnica en el Horizonte Tardío a partir de alfares definidos por los métodos convencionales y espectroscópicos (Makowski \& Vega-Centeno, 2004; Makowski et al., 2008). Para un estudio de procedencia con alcance regional, es recomendable tomar en cuenta ambas instancias, es decir, tanto la evaluación de la variabilidad interna de cada tipo cerámico como la localización de los centros de manufactura, pues una tipología sustentada por análisis composicional garantiza un muestreo adecuado. Para estudios limitados, es aceptable centrarse directamente en la procedencia, pero teniendo en cuenta las debilidades inherentes a una selección de muestras sin la evaluación composicional de la tipología cerámica.

Los métodos de análisis químico más usados en el estudio de la procedencia son la activación neutrónica (INAA), la espectrometría de fluorescencia de Rayos X (XRF), la espectrometría de masas por plasma inducido (ICP-MS), el análisis de emisión de Rayos X inducido por protones (PIXE), entre otros (Rye \& Duerden, 1982; Hatcher et al., 1995; Glascock et al., 2004; Stanjek \& Häusler, 2004). La selección de un método particular en el Perú dependerá del número de elementos que puede ser detectado, la exactitud y la precisión de los datos cuantitativos, la facilidad en la preparación y medición de la muestra y el costo.

La técnica de mayor uso y aceptación hasta la actualidad es INAA (Perlman \& Asaro, 1969; Harbottle, 1975; Glascock, 1992). Las ventajas principales de este método radican en su gran precisión, exactitud y sensibilidad en el análisis simultáneo de 30 o más elementos, y la facilidad con que se procesan muestras pequeñas de 50 hasta $200 \mathrm{mg}$. Una ventaja adicional es que cada serie de muestras analizada incluye en el conjunto la muestra estándar o de referencia, por lo que, al igual que otras técnicas, hay una compatibilidad demostrada entre los resultados de distintos laboratorios aplicando una simple fórmula de calibración. Se estima que se han analizado más de 100 mil muestras de cerámica y de arcilla en los laboratorios INAA en todo el mundo (Glascock et al., 2004). La activación neutrónica es tan ampliamente aceptada que se ha convertido en el estándar contra el que una nueva técnica se debe comparar. Una de las más recientes, LA-ICP-MS, ha alcanzado la madurez por su demostrada compatibilidad con los resultados de INAA (Larson et al., 2005; Speakman \& Neff, 2005a). Sin embargo, el análisis INAA toma 5 ó 6 semanas, es costoso y su aplicación depende de la disponibilidad de un reactor nuclear. La tendencia mundial actual parece orientarse a la desactivación de reactores de investigación, que son antiguos, se acercan al fin de su vida útil y no serían reemplazados (Glascock et al., 2004).

En el Perú hay un cierto retraso en la aplicación de INAA. Existen relativamente pocos estudios (D'Altroy et al., 1994; Chapdelaine et al., 1995; Vaughn \& Neff, 2000; Chapdelaine et al., 2001; Druc, 2004; Bray et al., 2005; Makowski \& Ghezzi, 2007; Vaughn \& Van Gijseghem, 2007). En los últimos años, el Instituto Peruano de Energía Nuclear (IPEN) ha estudiado cerámica arqueológica por INAA en su reactor de investigación de Huarangal (Delgado et al., 2007). En otras latitudes, la enorme cantidad de estudios INAA ya realizados genera cierta inercia para seguir usando, por razones institucionales y metodológicas, esta técnica, a 
pesar de su futuro incierto. Por lo tanto, la escasa aplicación a la cerámica del INAA en el Perú deja el camino libre para técnicas más modernas.

Es probable que ICP-MS sea la técnica que suceda a INAA, pues rivaliza con ella en sensibilidad y la supera en el número de elementos que mide. El ICP-MS puede reconocer virtualmente todos los elementos de la tabla periódica con buenos límites de detección. Además, mide proporciones de isótopos estables, que ayuda en la discriminación de fuentes de materia prima geoquímicamente similares. Cuando está acompañado de un sistema de evaporización o ablación por láser, o LA-ICPMS, solo toma minutos por muestra, cuesta poco (el $20 \%$ de un análisis INAA) y ocasiona daño mínimo al artefacto. Por estas razones, su uso en arqueología ha experimentado un gran impulso en años recientes (Gratuze et al., 2001; Kennett et al., 2002; Neff, 2002; Speakman \& Neff, 2002; Cecil, 2004; Kennett et al., 2004; Li et al., 2005; Speakman \& Neff, 2005b). En el Perú, la tecnología ICP-MS se encuentra disponible en los laboratorios ambientales, siendo posible usarla en aplicaciones arqueológicas.

\section{ANÁLISIS ESTADÍSTICO DE DATOS QUÍMICOS}

Debido a la variabilidad en la composición química de una fuente (y entre distintas fuentes) de materias primas, la determinación de la procedencia a partir de la composición requiere un número grande de muestras y el análisis simultáneo de 20 o más elementos químicos. Así, ante el número de variables involucrado (hasta 45 elementos con ICP-MS), se debe utilizar los métodos de exploración y clasificación de datos de la estadística multivariante.

Los datos composicionales son un espacio multidimensional, cada dimensión corresponde a la concentración de un elemento químico particular en cada muestra. Así, la muestra ocupa una posición única en el hiperespacio composicional: las de composición similar se ubican en posiciones cercanas entre sí, y las de composición diferente se ubican en posiciones distantes. Para definir un grupo composicional se debe identificar muestras cercanas entre sí. Cada grupo es un «centro de masa» en este hiperespacio, definido por las correlaciones entre las concentraciones de elementos y la posición del centro del grupo o «centroide» (Glascock et al., 2004).

Existen varios métodos estadísticos para identificar grupos composicionales: exploración de datos, componentes principales y agrupamiento (Tukey, 1977; Aitchison, 1986; Tyers \& Orton, 1991; Rattray \& Harbottle, 1992; Baxter, 1994; Beier \& Mommsen, 1994; Papageorgiou et al., 2001; Glascock et al., 2004). El paso inicial es la exploración de datos mediante diagramas de dispersión en dos y tres dimensiones, examinando correlaciones entre variables, identificando

1 Los procedimientos estadísticos mencionados en esta sección han sido compilados por MURR en una aplicación informática basada en la aplicación Gauss, disponible mediante solicitud en http:// archaeometry.missouri.edu, 
grupos composicionales obvios, detectando casos anormales, etc. Por ejemplo, la figura 2 es un diagrama de dispersión de concentraciones de elementos químicos en muestras de cerámica del valle de Lurín analizadas con LA-ICP-MS (Makowski et al., 2008). La proyección Cs-Rb discrimina efectivamente tres grupos composicionales principales.

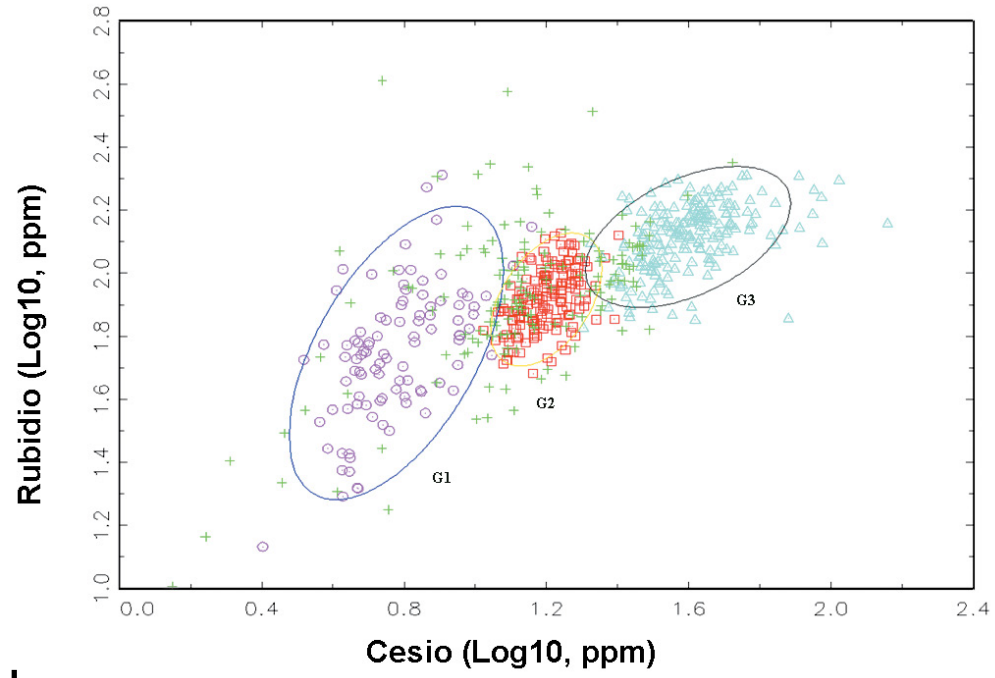

Figura 2 - Diagrama de dispersión de concentraciones de cesio y rubidio en los tres principales grupos composicionales

Las elipses representan los grupos a un nivel de confianza de $90 \%$ de pertenencia. El símbolo + representa las muestras no asignadas a ningún grupo. Los datos composicionales, en partes por millón (ppm), han sido transformados a logaritmos de base 10 (Makowski et al., 2008).

El análisis de agrupamiento mide la similitud entre pares de muestras y mediante un algoritmo de agrupamiento, crea un dendrograma en el que las muestras se ordenan de acuerdo a su similitud (Pérez López, 2004). Un problema con esta técnica es que sus variantes producen distintos resultados. Además, para el análisis se elige un número específico de elementos químicos, pero como los elementos que componen la cerámica tienden a tener una alta correlación entre sí, surgen complicaciones. Finalmente, los dendrogramas son difíciles de interpretar. Por lo general, el análisis de agrupamiento es solo útil para la definición de grupos hipotéticos a ser evaluados por otras técnicas multivariantes (Glascock et al., 2004).

El análisis de componentes principales es una técnica estadística de clasificación de datos muy empleada en el análisis de datos composicionales (Baxter, 1994; Pérez López, 2004). Mide la magnitud y dirección de las varianzas máximas de los datos en el hiperespacio composicional, y genera nuevas variables y valores a partir de las originales. Las nuevas variables, llamadas componentes principales, incorporan la mayor parte de la varianza en los datos originales. Los primeros 
componentes principales explican la mayor parte de la variabilidad entre las muestras; por lo tanto, un diagrama de dispersión permite la búsqueda visual de patrones composicionales (Baxter, 1994). La figura 3 es un gráfico de dispersión de componentes principales que muestra cuatro grupos composicionales en la muestra del valle de Lurín (Makowski et al., 2008). En este «biplot» (Baxter, 1992) se añadieron vectores al diagrama de dispersión, que conectan los elementos químicos con el punto de origen. Así se representa gráficamente la dirección y magnitud con que cada elemento químico contribuye a definir los grupos composicionales. Se observan dos conjuntos covariantes de elementos químicos, uno compuesto por los lantánidos, incluidos $\mathrm{Sn}$ y Th, y el otro compuesto por $\mathrm{Cs}, \mathrm{Sr}, \mathrm{K}, \mathrm{Zr}, \mathrm{Hf}, \mathrm{Sb}, \mathrm{Pb}, \mathrm{As}, \mathrm{Rb}, \mathrm{U}$, y Zn. Hay una baja correlación entre los dos conjuntos, indicada por el ángulo de aproximadamente $90^{\circ}$ entre los vectores. Mientras que los elementos del primer conjunto tienden a dispersar los grupos composicionales a lo largo de ejes paralelos, los elementos del segundo conjunto, más $\mathrm{Cu}$ y $\mathrm{Fe}$, los discriminan en forma óptima. Así, los últimos elementos pueden ser seleccionados como un subgrupo de variables para refinar los grupos composicionales en análisis posteriores.

Toda técnica estadística depende de ciertas premisas sobre la distribución de los datos que a veces no se cumplen. Para evitar el riesgo de «crear» grupos composicionales sin fundamento real, se debe realizar la verificación probabilística

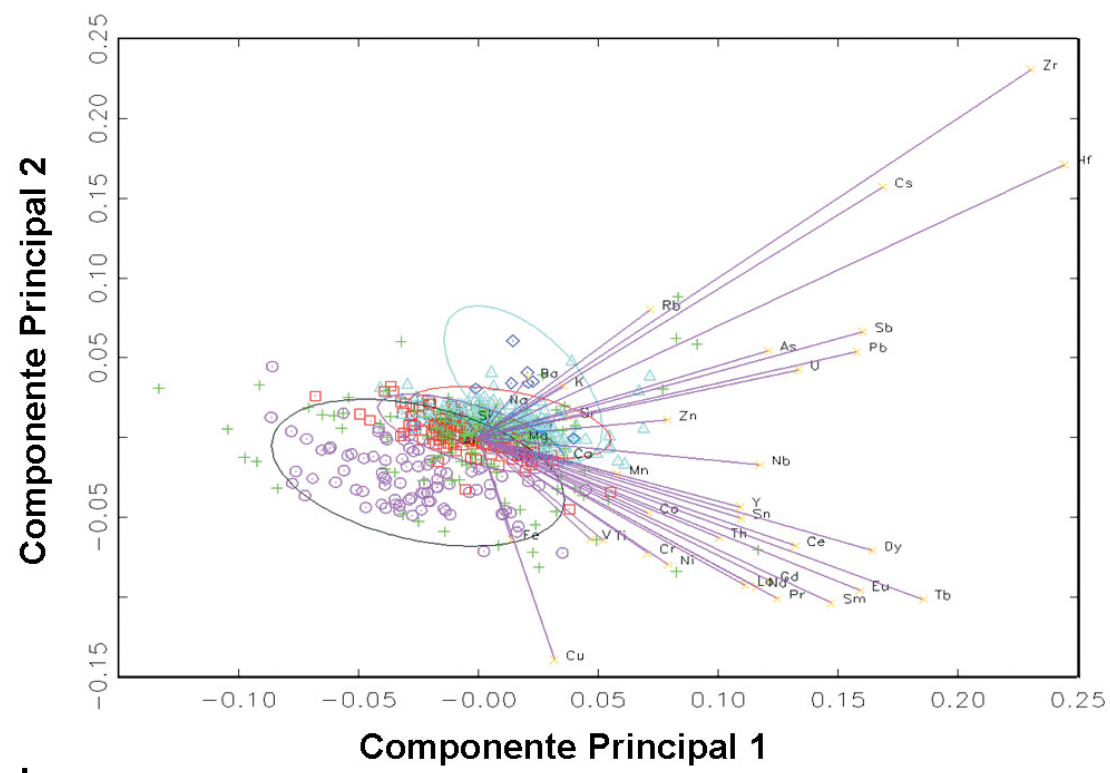

Figura 3 - «Biplot» (Baxter, 1992) de los componentes principales 1 y 2 de la matriz de varianza-covarianza, para los tres grupos composicionales principales

Las elipses representan los grupos a un nivel de confianza de $90 \%$ de pertenencia. Los vectores conectan las coordenadas de los elementos con el punto de origen. El símbolo + representa las muestras no asignadas a ningún grupo (Makowski et al., 2008). 
de la pertenencia de cada muestra a un grupo. El análisis de agrupamiento utiliza la distancia euclidiana para medir la similitud entre cada par de muestras, pero como ya se advirtiera, no considera las posibles correlaciones entre variables, algo que es común en cerámica. Por el contrario, la distancia Mahalanobis considera la varianza del grupo y la distancia entre su centro y la muestra, incorporando la correlación entre elementos composicionales. Así, permite evaluar la probabilidad estadística de pertenencia al grupo para cada muestra (Bishop \& Neff, 1989; Neff, 2000; Glascock, 2002; Glascock et al., 2004). Sin embargo, este enfoque ha sido criticado porque en algunos casos hasta un $30 \%$ de las muestras no resultan asignadas a ningún grupo (Stoltman et al., 2005).

Un método poco aplicado es el análisis discriminante, que evalúa grupos composicionales hipotéticos. Este método crea nuevas variables, o funciones discriminantes, que maximizan las distancias entre los grupos y minimizan las distancias internas (Pérez López, 2004). Luego, la probabilidad estadística de que la muestra pertenezca a un grupo hipotético se evalúa calculando la distancia Mahalanobis entre la muestra y el nuevo centro de cada grupo determinado por el análisis discriminante (Baxter, 1994). Makowski utiliza este método para mostrar la separación efectiva entre los tres grupos composicionales principales identificados en el valle de Lurín. El diagrama de dispersión en la figura 4 no revela ninguna muestra que, por su posición relativa al grupo asignado, deba ser removida o reclasificada (Makowski et al., 2008).

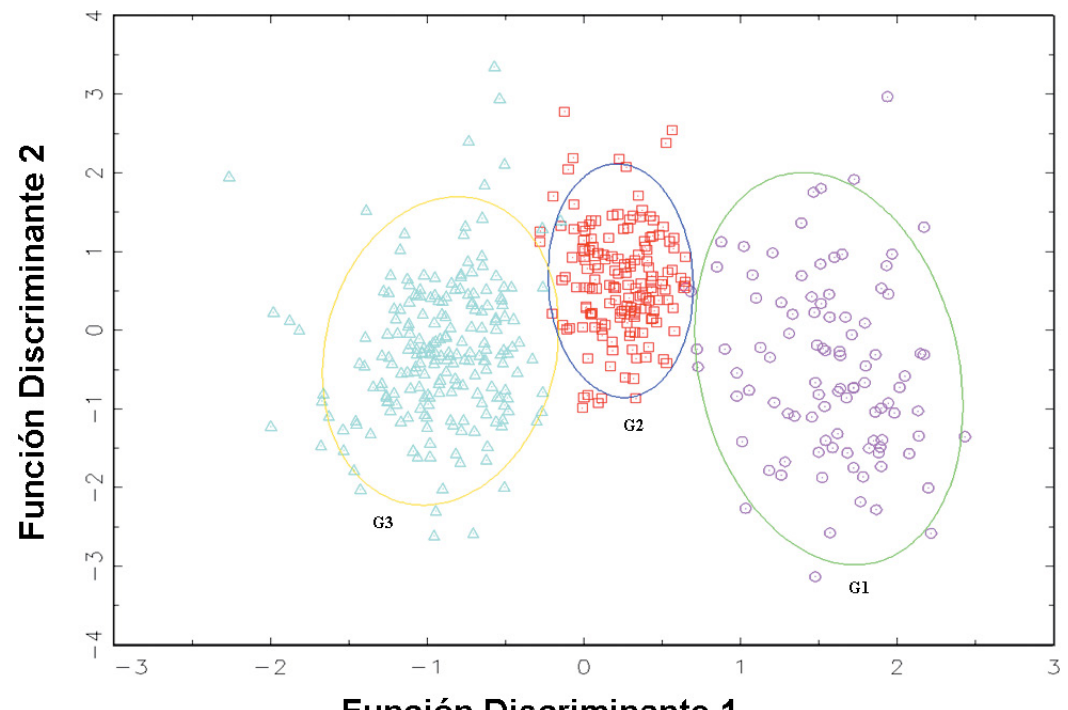

Función Discriminante 1

Figura 4 - Diagrama de dispersión de las funciones discriminantes en los grupos composicionales principales

Las elipses representan un nivel de confianza del $90 \%$ de pertenencia al grupo (Makowski et al., 2008). 


\section{LA INTERPRETACIÓN DE LOS RESULTADOS}

La interpretación de los datos implica contrastar los grupos composicionales con la procedencia arqueológica de la muestra, datos convencionales sobre alfares, formas, estilos, etc., y los datos geológicos (Tite, 1999; Mommsen, 2001; 2004).

Para cada grupo composicional, se debe establecer si fue producido en la localidad, si fue producido en la región inmediata e intercambiado, o si es un producto de intercambio de larga distancia. Para ello se debe identificar los centros de producción y/o las fuentes de materias primas (arcilla, inclusiones, pigmentos minerales). Naturalmente, esta interpretación es el paso más difícil en los estudios de procedencia, pues depende de la disponibilidad de material de referencia o comparativo, tanto geológico como arqueológico. En cuanto a los datos geológicos, se debe comparar la mineralogía y/o composición química de los grupos con la de las posibles fuentes identificadas en la región. Se debe buscar si las inclusiones en la cerámica pueden derivarse de la geología local, y si su composición química es consistente con las arcillas locales. Druc, por ejemplo, estudió la procedencia y circulación de cerámica de sitios del Horizonte Temprano mediante la comparación entre los datos de prospecciones geológicas y los resultados de análisis petrográfico y por fluorescencia de Rayos X (Druc, 1998).

En cuanto a la comparación arqueológica, los materiales de referencia ideales son los residuos de materia prima y los descartes de producción (recochos y otros), hallados en contexto en los centros de producción. Estos materiales generalmente no se trasladan ni intercambian, sino que se desechan en el lugar. Así, Chapdelaine utilizó como referencia para grupos composicionales definidos por activación neutrónica la arcilla en estado natural y/o en bolas hallada en las excavaciones de un taller de alfareros cercano a la huaca de la Luna (Chapdelaine et al., 1995). Sin embargo, los descartes de producción no siempre son tecnológicamente comparables con la cerámica que conforma el grupo composicional, pues pueden ser de otro tipo cerámico. Se debe tener cuidado en distinguir los desechos de producción de fragmentos similares que pueden ocurrir en contextos de consumo por su contacto con fuego a altas temperaturas. También hay que analizar los productos acabados y arcillas en estado natural de la región que abarca el centro de producción.

Cuando no se logra localizar directamente el centro de producción, se considera la distribución de frecuencias, espacial y tipológica del grupo composicional para identificar los lugares de procedencia de la cerámica. Así, el análisis cuantitativo de tipos cerámicos indicará si un patrón composicional es frecuente en un determinado lugar, pero poco común en otro; en este caso, podría haberse producido en el primer sitio. Del mismo modo, para vasijas de dimensiones y peso considerable, es razonable asumir que fueron fabricadas cerca de su lugar de hallazgo.

Si la distribución espacial y de frecuencias es parte de una tradición tecnológica, repitiéndose a través de varias fases cerámicas locales, la asignación del grupo composicional a la zona en la que se halló es confiable. Y si el grupo composicional 
incorpora piezas de tipos cerámicos diferentes, especialmente si se trata de alfares distintos, como piezas burdas y finas, es aún más probable que sea local. Por el contrario, cuando un grupo composicional presente en un sitio se halla sólo en cantidades inferiores a tipos comprobadamente locales, representa un alfar muy poco común, y además difiere química o mineralógicamente de los grupos composicionales o fuentes de materias primas locales, es razonable determinar que se trata de cerámica importada o «exótica».

Un obstáculo para la aplicación de análisis arqueométricos en cerámica prehispánica es la ausencia de material comparativo, lo que ocasiona grandes vacíos de información. En estos casos, la comparación entre las muestras y las fuentes geológicas de la materia prima debe tener prioridad sobre los criterios de distribución arqueológica en la definición de los centros de producción de la cerámica. Cuando la ausencia de información geológica obligue a dar mayor peso a los criterios de distribución arqueológica descritos previamente, estos deben usarse con cuidado para evitar razonamientos circulares. Sería erróneo asignar tentativamente un patrón composicional a una localidad determinada, en base a su abundancia, y en futuros estudios utilizar material del grupo composicional mencionado como referencia para esa localidad. Idealmente, un estudio integral, como los estudios a largo plazo en Conchucos (Druc, 1998; 2001; 2004), permite la definición de los centros de producción combinando la información geológica local con la distribución arqueológica de la cerámica e información etnoarqueológica.

\section{Agradecimientos}

Agradezco a Richard Burger (Universidad de Yale) y Krzysztof Makowski (Pontificia Universidad Católica del Perú, PUCP) por apoyar decisivamente desde un inicio mi interés por la arqueometría. Agradezco a Hector Neff (Instituto de Investigación Integrada en Materiales, Ambiente y Sociedad, IIRMES) y a Michael Glascock (Laboratorio de Arqueometría, Reactor Nuclear de Investigación, MURR), por su invalorable colaboración en proyectos conjuntos desde hace algunos años.

La Dirección Académica de Investigación PUCP (DAI), IIRMES, MURR, la Fundación Nacional de la Ciencia de Estados Unidos (NSF) y el Laboratorio de Microsonda Electrónica de la Universidad de Yale han financiado los estudios composicionales de cerámica del Proyecto Arqueológico Taller de Campo «Lomas de Lurín» (PATL). La sistematización de información y exhaustiva revisión bibliográfica para este artículo no hubieran sido posibles sin el apoyo continuo de todas estas instituciones.

\section{Referencias citadas}

ADAMS, W. Y. \& ADAMS, E. W., 1991 - Archaeological typology and practical reality: A dialectical approach to artifact classification and sorting, 427 pp.; Cambridge: Cambridge University Press.

AITCHISON, J., 1986 - The statistical analysis of compositional data, 416 pp.; New York: Chapman and Hall. 
ARNOLD, D. E., 1972 - Mineral analysis of ceramic materials from Quinua, department of Ayacucho, Peru. Archaeometry, 14: 93-102.

ARNOLD, D. E., 1993 - Ecology and ceramic production in an Andean community, 278 pp.; Cambridge: Cambridge University Press.

ARNOLD, D. E., 2000 - Does the standardization of ceramic pastes really mean specialization? Journal of Archaeological Method and Theory, 7 (4): 333-375.

BAXTER, M. J., 1992 - Archaeological uses of the biplot: A neglected technique? In: Computer Applications and Quantitative methods in Archaeology 1991 (G. Lock \& J. Moffett, eds.): 141-148; Oxford: B.A.R.

BAXTER, M. J., 1994 - Exploratory multivariate analysis in archaeology, 307 pp.; Edinburgh: Edinburgh University Press.

BEAUDRY, M. P., 1988 - Function of nonfunctional ceramics. In: Pot for all reasons: Ceramic ecology revisited (C. C. Kolb \& L. M. Lackey, eds.): 45-55; Philadelphia: Laboratory of Anthropology, Temple University.

BECK, M. E., SKIBO, J. M., HALLY, D. J. \& YANG, P., 2002 - Sample selection for ceramic use-alteration analysis: The effects of abrasion on soot. Journal of Archaeological Science, 29 (1): 1-15.

BEIER, T. \& MOMMSEN, H., 1994 - Modified Mahalanobis filters for grouping pottery by chemical composition. Archaeometry, 36 (2): 287-306.

BISHOP, R. L., 1980 - Aspects of ceramic compositional modeling. In: Models and methods in regional exchange (R. E. Fry, ed.): 47-65; Washington, D.C.: Society for American Archaeology.

BISHOP, R. L. \& NEFF, H., 1989 - Compositional data analysis in archaeology. In: Archaeological chemistry IV (R. O. Allen, ed.): 576-586; Washington, D.C.: American Chemical Society.

BISHOP, R. L., RANDS, R. L. \& HOLLEY, G. R., 1982 - Ceramic compositional analysis in archaeological perspective. Advances in Archaeological Method and Theory, 5: 275-330.

BOZARTH, S. R. \& GUDERJAN, T. H., 2004 - Biosilicate analysis of residue in Maya dedicatory cache vessels from Blue Creek, Belize. Journal of Archaeological Science, 31 (2): 205-215.

BRAY, T. L., MINC, L., CERUTI, M. C., CHÁVEZ, J. A., PEREA, R. \& REINHARD, J., 2005 - A compositional analysis of pottery vessels associated with the Inca ritual of Capacocha. Journal of Anthropological Archaeology, 24 (1): 82-100.

BROWN, L. D. \& HERON, C., 2003 - Boiling oil: The potential role of ceramics in recognising direct evidence for the exploitation of fish. In: Prehistoric pottery: People, pattern and purpose (A. M. Gibson, ed.): 35-42; Oxford: ArchaeoPress.

BUXEDA I GARRIGÓS, J., 1999 - Alteration and contamination of archaeological ceramics: The perturbation problem. Journal of Archaeological Science, 26 (3): 295-313.

CACKETTE, M., D'AURIA, J. M. \& SNOW, B. E., 1987 - Examining earthenware vessel function by elemental phosphorus content. Current Anthropology, 28 (1): 121-127.

CALEY, E. R., 1967 - The early history of chemistry in the service of archaeology. Journal of Chemical Education, 44 (3): 120.

CARR, C., 1990 - Advances in ceramic radiography and analysis: Applications and potentials. Journal of Archaeological Science, 17 (1): 13-34.

CECIL, L. G., 2004 - Inductively coupled plasma emission spectroscopy and postclassic Petén slipped pottery: An examination of pottery wares, social identity and trade. Archaeometry, 46 (3): 385-404. 
CHAPDELAINE, C., KENNEDY, G. \& UCEDA CASTILLO, S., 1995 - Activación neutrónica en el estudio de la producción local de la cerámica ritual en el sitio Moche, Perú. Bulletin de l'Institut Français d'Études Andines, 24 (2): 183-212.

CHAPDELAINE, C., MILLAIRE, J.-F. \& KENNEDY, G., 2001 - Compositional analysis and provenance study of spindle whorls from the Moche site, north coast of Peru. Journal of Archaeological Science, 28 (8): 795-806.

CHAPDELAINE, C., MINEAU, R. \& UCEDA CASTILLO, S., 1997 - Estudio de los pigmentos de la cerámica ceremonial Moche con ayuda de un microscopio electrónico de barrido. Bulletin de I'Institut Français d'Études Andines, 26 (2): 229-245.

CHAPDELAINE, C., PIMENTEL, V. \& GAMBOA, J., 2009 - Rol de la cerámica en la afirmación social de los Moche del valle de Santa. Revista Chilena de Antropología, 20: $37-76$.

CHARTERS, S., EVERSHED, R. P., QUYE, A., BLINKHORN, P. W. \& REEVES, V., 1997 Simulation experiments for determining the use of ancient pottery vessels: The behaviour of epicuticular leaf wax during boiling of a leafy vegetable. Journal of Archaeological Science, 24 (1): 1-7.

COGSWELL, J. W., NEFF, H. \& GLASCOCK, M. D., 1996 - Effect of firing temperature on the elemental characterization of pottery. Journal of Archaeological Science, 23 (2): 283-287.

COSTIN, C. L., 2001 - Craft production systems. In: Archaeology at the millennium: A sourcebook: 273-327; New York: Kluwer Academic/Plenum.

CROWN, P. L. \& BISHOP, R. L., 1994 - Ceramics and ideology: Salado polychrome pottery, 254 pp.; Albuquerque: University of New Mexico Press.

D'ALTROY, T. N. \& BISHOP, R. L., 1990 - The provincial organization of Inka ceramic production. American Antiquity, 55 (1): 120-138.

D'ALTROY, T. N., LORANDI, A. M. \& WILLIAMS, V., 1994 - Producción y uso de cerámica en la economía política Inka. In: Tecnología y organización de la producción cerámica prehispánica en los Andes (I. Shimada, ed.): 395-442; Lima: Pontificia Universidad Católica del Perú.

DELGADO, M., OLIVERA, P., MONTOYA, E. \& BUSTAMANTE, A., 2007 - Building a bridge to the past: Archaeometry at the IPEN reactor. Archaeometry, 49 (2): 403-412.

DESCHLER-ERB, E., LEHMANN, E. H., PERNET, L., VONTOBEL, P. \& HARTMANN, S., 2004 - The complementary use of neutrons and x-rays for the non-destructive investigation of archaeological objects from Swiss collections. Archaeometry, 46 (4): 647-661.

DRUC, I. C., 1998 - Ceramic production and distribution in the Chavin sphere of influence (north-central Andes), 121 pp.; Oxford: J. and E. Hedges.

DRUC, I. C., 2001 - ¿Shashal o no shashal?, esa es la cuestión. Etnoarqueología cerámica en la zona de Huari, Ancash. Bulletin de l'Institut Français d'Études Andines, 30 (1): $1-17$.

DRUC, I. C., 2004 - Ceramic diversity in Chavín de Huantar, Peru. Latin American Antiquity, 15 (3): 344-363.

DRUC, I. C., BURGER, R. L., ZAMOJSKA, R. \& MAGNY, P., 2001 - Ancón and Garagay ceramic production at the time of Chavín de Huántar. Journal of Archaeological Science, 28 (1): 29-43.

DUDD, S. N., EVERSHED, R. P. \& GIBSON, A. M., 1999 - Evidence for varying patterns of exploitation of animal products in different prehistoric pottery traditions based on lipids preserved in surface and absorbed residues. Journal of Archaeological Science, 26 (12): 1473-1482. 
DUNNELL, R. C. \& HUNT, T. L., 1990 - Elemental composition and inference of ceramic vessel function. Current Anthropology, 31 (3): 330-336.

EERKENS, J. W. \& BETTINGER, R. L., 2001 - Techniques for assessing standardization in artifact assemblages: Can we scale material variability? American Antiquity, 66 (3): 493-504.

EVERSHED, R. P., CHARTERS, S. \& QUYE, A., 1995 - Interpreting lipid residues in archaeological ceramics: Preliminary results from laboratory simulations of vessel use and burial. In: Materials issues in art and archaeology iv (P. B. Vandiver, J. R. Druzik, J. L. Galván, G. S. Wheeler \& I. C. Freestone, eds.): 85-95; Pittsburgh: Materials Research Society.

EVERSHED, R. P., VAUGHAN, S. J., DUDD, S. N. \& SOLES, J. S., 2000 - Organic residue, petrographic and typological analyses of late Minoan lamps and conical cups from excavations at Mochlos in East Crete, Greece. In: Paleodiet in the Aegean (S. J. Vaughan \& W. D. E. Coulson, eds.): 37-54; Oxford: Oxbow.

FRANKLIN, U. M. \& VITALI, V., 1985 - Environmental stability of ancient ceramics. Archaeometry, 27 (1): 3-15.

FREESTONE, I. G. \& MIDDLETON, A. P., 1987 - Mineralogical applications of the analytical SEM in archaeology. Mineralogical Magazine, 51(1): 21-31.

FROH, J., 2004 - Archaeological ceramics studied by scanning electron microscopy. Hyperfine Interactions, 154: 159-176.

GARCÍA-HERAS, M., BLACKMAN, M. J., FERNÁNDEZ-RUIZ, R. \& BISHOP, R. L., 2001 Assessing ceramic compositional data: A comparison of total reflection x-ray fluorescence and instrumental neutron activation analysis on late iron age Celtiberian ceramics. Archaeometry, 43 (3): 325-347.

GLASCOCK, M. D., 1992 - Characterization of archaeological ceramics at MURR by neutron activation analysis and multivariate statistics. In: Chemical characterization of ceramic pastes in archaeology (H. Neff, ed.): 11-26; Madison: Prehistory Press.

GLASCOCK, M. D., 2002 - Geochemical evidence for long-distance exchange, 282 pp.; Westport: Bergin and Garvey.

GLASCOCK, M. D., NEFF, H. \& VAUGHN, K. J., 2004 - Instrumental neutron activation analysis and multivariate statistics for pottery provenance. Hyperfine Interactions, 154: 95-105.

GOSSELAIN, O. P., 1992 - Technology and style: Potters and pottery among Bafia of Cameroon. Man, 27 (3): 559-586.

GRATUZE, B., BLET-LEMARQUAND, M. \& BARRANDON, J. N., 2001 - Mass spectrometry with laser sampling: A new tool to characterize archaeological materials. Journal of Radioanalytical and Nuclear Chemistry, 247 (3): 645 - 656.

GUFFROY, J. (ed.), 1994 - Cerro ñañañique: Un établissement monumental de la période formative, en limite de désert (Haut Piura, Pérou), 440 pp.; París: ORSTOM.

HALLY, D. J., 1983 - Use alteration of pottery vessel surfaces: An important source of evidence for the identification of vessel function. North American Archaeologist, 4 (1): 3-26.

HALLY, D. J., 1986 - Identification of vessel function: A case study from northwest Georgia. American Antiquity, 51 (2): 267-295.

HANKEY, V., 1983 - Ceramic tradition in late bronze age Cyprus. Report of the Department of Antiquities, Cyprus: 168-171.

HARBOTTLE, G., 1975 - Activation analysis study of ceramics from the Capacha (Colima) and Opeño (Michoacán) phases of west Mexico. American Antiquity, 40 (4): 453-458. 
HARBOTTLE, G., 1991 - The efficiencies and error-rates of Euclidian and Mahalanobis searches in hypergeometries of archaeological ceramic composition. In: Archaeometry '90 (E. Pernicka \& G. A. Wagner, eds.): 413-424; Berlin: Birkhäuser Verlag.

HATCHER, H., WALSH, J. N. \& TITE, M. S., 1995 - Comparison of inductively-coupled plasma emission spectrometry and atomic absorption spectrometry analysis on standard reference silicate materials and ceramics. Archaeometry, 37 (1): 83-94.

HAYASHIDA, F., GLASCOCK, M., HÄUSLER, W., NEFF, H., RIEDERER, J. \& WAGNER, U., 2002 - Technology and organization of Inka pottery production: Archaeometric perspectives. In: Archaeometry 98: Proceedings of the 31st symposium, Budapest, april 26-may 3, 1998 (E. Jerem, K. T. Biró \& E. Rudner, eds.): 573-580; Oxford: Archaeopress.

HENRICKSON, E. F. \& MCDONALD, M. M. A., 1983 - Ceramic form and function: An ethnographic search and an archaeological application. American Anthropologist, 85 (3): 630-643.

HERON, C. \& EVERSHED, R. P., 1993 - Analysis of organic residues and the study of pottery use. Archaeological Method and Theory, 5: 247-284.

KENNETT, D. J., ANDERSON, A. J., CRUZ, M. J., CLARK, G. R. \& SUMMERHAYES, G. R., 2004 - Geochemical characterization of Lapita pottery via inductively coupled plasma-mass spectrometry (ICP-MS). Archaeometry, 46 (1): 35-46.

KENNETT, D. J., SAKAI, S., NEFF, H., GOSSETT, R. \& LARSON, D. O., 2002 - Compositional characterization of prehistoric ceramics: A new approach. Journal of Archaeological Science, 29 (5): 443-455.

KILIKOGLOU, V. \& VEKINIS, G., 2002 - Failure prediction and function determination of archaeological pottery by finite element analysis. Journal of Archaeological Science, 29 (11): 1317-1325.

KILIKOGLOU, V., MANIATIS, Y. \& GRIMANIS, A. P., 1988 - Effect of purification and firing of clays on trace element provenance studies. Archaeometry, 30 (1): 37-46.

KOBAYASHI, M., 1994 - Use-alteration analysis of Kalinga pottery: Interior carbon deposits of cooking pots. In: Kalinga ethnoarchaeology: Expanding archaeological method and theory (W. A. Longacre \& J. M. Skibo, eds.): 127-168; Washington, D.C.: Smithsonian Institution Press.

LARSON, D. O., SAKAI, S. \& NEFF, H., 2005 - Laser ablation inductively coupled plasma mass spectrometry (LA-ICP-MS) as a bulk chemical characterization technique: Comparison of LA-ICP-MS, digestion-ICP-MS, and INAA data on Virgin Branch Anasazi ceramics. In: Laser ablation ICP-MS in archaeological research (R. J. Speakman \& H. Neff, eds.): 95-102; Albuquerque: University of New Mexico Press.

LEEUW, S. E. V. D. \& PRITCHARD, A. C., 1984 - The many dimensions of pottery: Ceramics in archaeology and anthropology, 797 pp.; Amsterdam: Universiteit van Amsterdam.

LEMOINE, C. \& PICON, M., 1982 - Fixation du phosphore par les céramiques lors de leur enfouissement et ses incidences analytiques. Revue d'Archéométrie, 6: 101-112.

LI, B.-P., GREIG, A., ZHAO, J.-X., COLLERSON, K. D., QUAN, K.-S., MENG, Y.-H. \& MA, Z.L., 2005 - ICP-MS trace element analysis of song dynasty porcelains from Ding, Jiexiu and Guantai kilns, north China. Journal of Archaeological Science, 32: 251-259.

LUMBRERAS, L. G., 2005 - Arqueología y sociedad, 320 pp.; Lima: IEP.

LUMBRERAS, L. G., GEBHARD, R., HÄUSLER, W., SIEBEN, G., WAGNER, U., KAUFFMANNDOIG, F. \& RIEDERER, J., 2003 - Mössbauer study of ceramic finds from the Galeria de las Ofrendas, Chavín de Huantar. Hyperfine Interactions, 150 (1-4): 51-72. 
MAGGETTI, M., 1982 - Phase analysis and its significance for technology and origin. In: Archaeological ceramics (J. S. Olin \& A. D. Franklin, eds.): 121-133; Washington D.C.: Smithsonian Institution Press.

MAKOWSKI, K., 2002 - Power and social ranking at the end of the formative period: The lower Lurín valley cemeteries. In: Andean archaeology I: Variations in sociopolitical organization (W. H. Isbell \& H. Silverman, eds.): 89-120; New York: Kluwer Academic/Plenum.

MAKOWSKI, K. \& GHEZZI, I., 2007 - Reconstrucción de las redes de producción e intercambio en el Horizonte Tardío a través de la caracterización arqueométrica de las principales fuentes de arcilla y estilos cerámicos en las cuencas de los ríos Lurín, Rimac, y Chillón, Perú; Lima: PUCP. Proyecto de Investigación financiado por Dirección Académica de Investigación.

MAKOWSKI, K. \& VEGA-CENTENO, M., 2004 - Estilos regionales en la costa central en el Horizonte Tardío: Una aproximación desde el valle de Lurín. Bulletin de I'Institut Français d'Études Andines, 33 (3): 681-714.

MAKOWSKI, K., GHEZZI, I., GUERRERO, D., NEFF, H., JIMÉNEZ, M., ORE, G., \& ÁLVAREZCALDERÓN, R., 2008 - Pachacamac, Ychsma y los Caringas: Estilos e identidades en el valle de Lurín inca. In: Arqueología de la costa centro sur peruana (O. Pinedo \& H. Tantaleán, eds.): 267-316; Lima: Avqi ediciones.

MIDDLETON, A. P., 2005 - Ceramics. In: Radiography of cultural material (J. Lang \& A. Middleton, eds.): 60-81; Oxford: Butterworth, Heinemann.

MILLS, B. J., 1989 - Integrating functional analyses of vessels and sherds through models of ceramic assemblage formation. World Archaeology, 21 (1): 133-147.

MOMMSEN, H., 2001 - Provenance determination of pottery by trace element analysis: Problems, solutions and applications. Journal of Radioanalytical and Nuclear Chemistry, 247 (3): 657-662.

MOMMSEN, H., 2004 - Short note: Provenancing of pottery-the need for an integrated approach? Archaeometry, 46 (2): 267-271.

MOMMSEN, H., BEIER, T. \& WEBER, J., 1991 - Precision of neutron activation analysis in archaeometric studies of ceramics. In: Applications of nuclear techniques (G. Vourvopoulos \& T. Paradellis, eds.): 197-201; Singapore: World Scientific.

MONTOYA, E., ZAPATA, J., MENDOZA, P. \& GLOWACKI, M., 2003 - Chemical characterization of archaeological ceramics using k0-based instrumental neutron activation analysis: A study in the production and distribution of Middle Horizon pottery of Cuzco, Peru; Lima: Reporte Técnico presentado al Instituto Peruano de Energia Nuclear.

NEFF, H., 2000 - Neutron activation analysis for provenance determination in archaeology. In: Modern analytical methods in art and archaeology (E. Ciliberto \& G. Spoto, eds.): 81-134; New York: Wiley.

NEFF, H., 2002 - Analysis of Mesoamerican Plumbate pottery surfaces by laser ablation-inductively coupled plasma-mass spectrometry (LA-ICP-MS). Journal of Archaeological Science, 30 (1): 21-35.

NEFF, H., BLOMSTER, J. P., GLASCOCK, M. D., BISHOP, R. L., BLACKMAN, M. J., COE, M. D., COWGILL, G. L., CYPHERS, A., DIEHL, R. A., HOUSTON, S., JOYCE, A. A., LIPO, C. P. \& WINTER, M., 2006a - Smokescreens in the provenance investigation of Early Formative Mesoamerican ceramics. Latin American Antiquity, 17 (1): 104-118.

NEFF, H., BLOMSTER, J. P., GLASCOCK, M. D., BISHOP, R. L., BLACKMAN, M. J., COE, M. D., COWGILL, G. L., DIEHL, R. A., HOUSTON, S., JOYCE, A. A., LIPO, C. P., STARK, B. L. \& WINTER, M., 2006b - Methodological issues in the provenance investigation of Early Formative Mesoamerican ceramics. Latin American Antiquity, 17 (1): 54-76. 
NEUPERT, M. A., 1994 - Strength testing archaeological ceramics: A new perspective. American Antiquity, 59 (4): 709-723.

ORTON, C., 1993 - How many pots make five?: An historical review of pottery quantification. Archaeometry, 35 (2): 169-184.

ORTON, C., TYERS, P. \& VINCE, A. G., 1993 - Pottery in archaeology, 269 pp.; Cambridge: Cambridge University Press.

PAPAGEORGIOU, I., BAXTER, M. J. \& CAU, M. A., 2001 - Model-based cluster analysis of artefact compositional data. Archaeometry, 43 (4): 571-588.

PEACOCK, D. P. S., 1982 - Pottery in the Roman world: An ethnoarchaeological approach, 192 p.; London: Longman.

PÉREZ LÓPEZ, C., 2004 - Técnicas de análisis multivariante de datos: Aplicaciones con SPSS, 646 p.; Madrid: Pearson Educación.

PERLMAN, I. \& ASARO, F., 1969 - Pottery analysis by neutron activation. Archaeometry, 11: 21-52.

PILLACA, M., LANDAURO, C. V., LAZO, R. \& MAC KAY, M., 2009 - Caracterización de cerámica pre-colombina de estilo Chakipampa y Lima. Revista de Investigación de Física, 12 (2): 1-5.

PICON, M., 1987 - Fixation du baryum et du strontium par les céramiques. Revue d'Archéometrie, (11): 41-47.

POLVORINOS DEL RÍO, A., GARCÍA SANJUÁN, L., HURTADO PÉREZ, V. \& HERNÁNDEZ ARNEDO, M. J., 2005 - Bronze age ceramics in southwest Spain: An exploratory archaeometric study of technology and function. Geoarchaeology, 20 (3): 263-284.

POOL, C. A., 1992 - Integrating ceramic production and distribution. In: Ceramic production and distribution: An integrated approach (G. J. Bey \& C. A. Pool, eds.): 275-313; Boulder: Westview Press.

PURIN, S., 1985 - Construction de trois vases noirs Mochicas. Bulletin des Musées Royaux d'Art et d'Histoire, 56 (1): 95-104.

RATTRAY, E. C. \& HARBOTTLE, G., 1992 - Neutron activation analysis and numerical taxonomy of thin orange ceramics from the manufacturing sites of Rio Carnero, Puebla, Mexico. In: Chemical characterization of ceramic pastes in archaeology (H. Neff, ed.): 221-231; Madison: Prehistory Press.

REBER, E. A., DUDD, S. N., VAN DER MERWE, N. J. \& EVERSHED, R. P., 2004 - Direct detection of maize in pottery residues via compound specific stable carbon isotope analysis. Antiquity, 78 (301): 682-691.

REGERT, M., VACHER, S., MOULHERAT, C. \& DECAVALLAS, O., 2003 - Adhesive production and pottery function during the iron age at the site of Grand Aunay (Sarthe, France). Archaeometry, 45 (1): 101-120.

RICE, P. M., 1987 - Pottery analysis: A sourcebook, 559 pp.; Chicago: University of Chicago Press.

RICE, P. M., 1991 - Specialization, standardization, and diversity: A retrospective. In: Ceramic legacy of Anna O. Shepard (R. L. Bishop \& F. W. Lange, eds.): 257-279; Niwot: University Press of Colorado.

RICE, P. M., 1996 - Recent ceramic analysis: 1, function, style, and origins. Journal of Archaeological Research, 4 (2): 133-163.

RIEDERER, J., 2004 - Thin section microscopy applied to the study of archaeological ceramics. Hyperfine Interactions, 154: 143-158.

ROHFRITSCH, A., 2006 - Céramiques Mochicas de la vallée de Jequetepeque (Pérou). Étude technique et physicochimique d'exemplaires provenant de Dos Cabezas et de San José de Moro; Bordeaux: Université Michel de Montaigne Bordeaux 3. Tesis de Maestría 2, Archéomatériaux. 
ROHFRITSCH, A., 2007 - La producción de cerámica fina mochica en el valle de Jequetepeque: Enfoque tecnológico, fisico-químico y experimental; Lima. Programa Arqueológico San José de Moro: Informe de Excavaciones Temporada 2006.

ROUMPOU, M., HERON, C., ANDREOU, S. \& KOTSAKIS, K., 2003 - Organic residues in storage vessels from the Toumba Thessalonikis. In: Prehistoric pottery: People, pattern and purpose (A. M. Gibson, ed.): 189-199; Oxford: ArchaeoPress.

ROUX, V., 2003 - Ceramic standardization and intensity of production: Quantifying degrees of specialization. American Antiquity, 68 (4): 768-782.

ROZENBERG, C. \& PICON, M., 1990 - Circulation, échange et production de poteries dans les Andes centrales au deuxième millénaire avant J. C. Bulletin de l'Institut Français d'Études Andines, 19 (1): 1-14.

RYE, O. S., 1981 - Pottery technology, 150 pp.; Washington, D.C.: Taraxacum.

RYE, O. S. \& DUERDEN, P., 1982 - Papuan pottery sourcing by PIXE: Preliminary studies. Archaeometry, 24 (1): 59-64.

SALAZAR, R., WAGNER, U., WAGNER, F. E., KORSCHINSKY, W., ZAHN, M., RIEDERER, J. \& KAUFFMANN-DOIG, F., 1986 - Mössbauer spectroscopy and neutron activation analysis of recent finds from Chavin. In: Proceedings of the 24th international archaeometry symposium (J. S. Olin \& M. J. Blackman, eds.): 143-151; Washington D.C.: Smithsonian Institution Press.

SAUNDERS, R. \& HAYS, C. T., 2004 - Early pottery: Technology, function, style, and interaction in the lower Southeast, 276 pp.; Tuscaloosa: University of Alabama Press.

SAYRE, E., DODSON, R. W. \& THOMPSON, D. B., 1957 - Neutron activation study of Mediterranean potsherds. American Journal of Archaeology, 61: 35-41.

SAYRE, E. V., 1988 - Application of compositional analysis to the study of materials and objects of art and archaeology. In: Materials issues in art and archaeology (E. V. Sayre, P. B. Vandiver, J. Druzik \& C. Stevenson, eds.): 41-51; Pittsburgh: Materials Research Society.

SCHIFFER, M. B., 1989 - Research design for ceramic use-wear analysis at Grasshopper Pueblo. In: Pottery technology: Ideas and approaches (G. Bronitsky, ed.): 183-205; Boulder: Westview Press.

SCHIFFER, M. B., 1996 - Formation processes of the archaeological record, 428 pp.; Salt Lake City: University of Utah Press.

SCHWEDT, A., MOMMSEN, H. \& ZACHARIAS, N., 2004 - Post-depositional elemental alterations in pottery: Neutron activation analyses of surface and core samples. Archaeometry, 46 (1): 85-101.

SEGURA, R., 2001 - Rito y economía en Cajamarquilla: Investigaciones arqueológicas en el conjunto arquitectónico Julio C. Tello, 203 pp.; Lima: Fondo Editorial de la Pontificia Universidad Católica del Perú.

SHARER, R. J., BALKANSKY, A. K., BURTON, J. H., FEINMAN, G. M., FLANNERY, K. V., GROVE, D. C., MARCUS, J., MOYLE, R. G., PRICE, T. D., REDMOND, E. M., REYNOLDS, R. G., RICE, P. M., SPENCER, C. S., STOLTMAN, J. B. \& YAEGER, J., 2006 - On the logic of archaeological inference: Early Formative pottery and the evolution of Mesoamerican societies. Latin American Antiquity, 17 (1): 90-103.

SHIMADA, I. (ed.), 1994 - Tecnología y organización de la producción cerámica prehispánica en los Andes, 517 pp.; Lima: Fondo Editorial de la Pontificia Universidad Católica del Perú.

SHIMADA, I., GOLDSTEIN, D., SOSA, J. \& WAGNER, U., 2003a - Early pottery making in northern coastal Peru. Part II: Field firing experiments. Hyperfine Interactions, 150 (1-4): 91-105. 
SHIMADA, I., HÄUSLER, W., HUTZELMANN, T., WAGNER, U. \& RIEDERER, J., 2003b Early pottery making in northern coastal Peru. Part III: Mössbauer study of Sican pottery. Hyperfine Interactions, 150 (1-4): 107-123.

SHIMADA, I., ELERA, C. G., CHANG, V., NEFF, H., GLASCOCK, M. D., WAGNER, U. \& GEBHARD, R., 1994 - Hornos y producción de cerámica durante el Periodo Formativo en Batán Grande, costa norte del Perú. In: Tecnología y organización de la producción cerámica prehispánica en los Andes (I. Shimada, ed.): 67-120; Lima: Fondo Editorial de la Pontificia Universidad Católica del Perú.

SKIBO, J. M., 1992 - Pottery function: A use-alteration perspective, 205 pp.; New York: Plenum Press.

SMITH, G. D. \& CLARK, R. J. H., 2004 - Raman microscopy in archaeological science. Journal of Archaeological Science, 31(8): 1137-1160.

SPEAKMAN, R. J. \& NEFF, H., 2002 - Evaluation of painted pottery from the Mesa Verde region using laser ablation - inductively coupled plasma-mass spectrometry (LA-ICPMS). American Antiquity, 67 (1): 137-144.

SPEAKMAN, R. J. \& NEFF, H., 2005a - The application of laser ablation ICP-MS to the study of archaeological materials: An introduction. In: Laser ablation ICP-MS in archaeological research (R. J. Speakman \& H. Neff, eds.): 1-14; Albuquerque: University of New Mexico Press.

SPEAKMAN, R. J. \& NEFF, H., 2005b - Laser ablation ICP-MS in archaeological research, 200 pp.; Albuquerque: University of New Mexico Press.

STALLER, J. E. \& THOMPSON, R. G., 2002 - A multidisciplinary approach to understanding the initial introduction of maize into coastal Ecuador. Journal of Archaeological Science, 29 (1): 33-50.

STANJEK, H. \& HÄUSLER, W., 2004 - Basics of x-ray diffraction. Hyperfine Interactions, 154: 107-119.

STOLTMAN, J. B., BURTON, J. H. \& HAAS, J., 1992 - Chemical and petrographic characterizations of ceramic pastes in archaeology: Two perspectives on a single data set. In: Chemical characterization of pastes in archaeology (H. Neff, ed.): 85-92; Madison: Prehistory Press.

STOLTMAN, J. B. \& MAINFORT, R. C., 2002 - Minerals and elements: Using petrography to reconsider the findings of neutron activation analysis of ceramics from Pinson mounds, Tennessee. Midcontinental Journal of Archaeology, 27 (1): 1-33.

STOLTMAN, J. B., BURTON, J. H., MARCUS, J., FLANNERY, K. V. \& MOYLE, R. G., 2005 Petrographic evidence shows that pottery exchange between the Olmec and their neighbors was two-way. Proceedings of the National Academy of Sciences of the United States of America, 102 (32): 11213-11218.

STOREY, J. M. V., 1988 - Chemical study of clays and roman pottery from the lower Nene valley, eastern England. Journal of Archaeological Science, 15 (1): 35-50.

THIRIET, C., 2008 - Les céramiques Cajamarca du site de San José de Moro: Étude comparative des groupes stylistiques «serrano» et «costeño»; Bordeaux: Université Michel de Montaigne Bordeaux 3. Tesis de Maestría 2, Archéomatériaux.

TITE, M. S., 1982 - The use of scanning electron microscopy in the technological examination of ancient ceramics. In: Archaeological ceramics (J. S. Olin \& A. D. Franklin, eds.): 109-120; Washington D.C.: Smithsonian Institution Press.

TITE, M. S., 1999 - Pottery production, distribution, and consumption. The contribution of the physical sciences. Journal of Archaeological Method and Theory, 6 (3): 181-233.

TITE, M. S., KILIKOGLOU, V. \& VEKINIS, G., 2001 - Strength, toughness and thermal shock resistance of ancient ceramics, and their influence on technological choice. Archaeometry, 43 (3): 301-324. 
TSCHAUNER, H. \& WAGNER, U., 2003 - Pottery from a Chimú workshop studied by Mössbauer spectroscopy. Hyperfine Interactions, 150 (1-4): 165-186.

TUKEY, J. W., 1977 - Exploratory data analysis, 688 pp.; Reading: Addison-Wesley.

TYERS, P. \& ORTON, C., 1991 - Statistical analysis of ceramic assemblages a year's progress. In: Computer applications and quantitative methods in archaeology 1990 (K. Lockyear \& S. P. Q. Rahtz, eds.): 117-120; Oxford: B.A.R.

UNDERHILL, A. P., 2003 - Investigating variation in organization of ceramic production: An ethnoarchaeological study in Guizhou, China. Journal of Archaeological Method and Theory, 10 (3): 203-275.

VAUGHN, K. J., CONLEE, C. A., NEFF, H. \& SCHREIBER, K., 2006 - Ceramic production in ancient Nasca: Provenance analysis of pottery from the early Nasca and Tiza cultures through INAA. Journal of Archaeological Science, 33 (5): 681-689.

VAUGHN, K. J., CONLEE, C. A., NEFF, H. \& SCHREIBER, K. J., 2005 - A compositional analysis of Nasca pigments: Implications for craft production on the pre-hispanic south coast of Peru. In: Laser ablation ICP-MS in archaeological research (R. J. Speakman \& H. Neff, eds.): 139-153; Albuquerque: University of New Mexico Press.

VAUGHN, K. J. \& NEFF, H., 2000 - Moving beyond iconography: Neutron activation analysis of ceramics from Marcaya, Peru, an early Nasca domestic site. Journal of Field Archaeology, 27 (1): 75-90.

VAUGHN, K. J. \& NEFF, H., 2004 - Tracing the clay source of Nasca polychrome pottery: Results from a preliminary raw material survey. Journal of Archaeological Science, 31 (11): 1577-1586.

VAUGHN, K. J. \& VAN GIJSEGHEM, H., 2007 - A compositional perspective on the origins of the «Nasca cult» at cahuachi. Journal of Archaeological Science, 34 (5): 814-822.

WAGNER, F. E. \& KYEK, A., 2004 - Mössbauer spectroscopy in archaeology: Introduction and experimental considerations. Hyperfine Interactions, 154: 5-33.

WAGNER, U., HÄUSLER, W., WAGNER, F. E. \& SHIMADA, I., 2003 - Mössbauer spectroscopy in South American archaeology. Hyperfine Interactions, 148-149 (1-4): 13-20.

WAGNER, U., WAGNER, F. E., HÄUSLER, W. \& SHIMADA, I., 2000 - The use of Mössbauer spectroscopy in studies of archaeological ceramics. In: Radiation in art and archaeometry (D. C. Creagh \& D. A. Bradley, eds.): 417-443; Amsterdam: Elsevier Science.

WEERD, J. V. D., SMITH, G. D., FIRTH, S. \& CLARK, R. J. H., 2004 - Identification of black pigments on prehistoric southwest American potsherds by infrared and Raman microscopy. Journal of Archaeological Science, 31 (10): 1429-1437.

WEIGAND, P. C., HARBOTTLE, G. \& SAYRE, E. V., 1977 - Turquoise sources and source analysis: Mesoamerica and the southwestern U.S.A. In: Exchange systems in prehistory (T. K. Earle \& J. E. Ericson, eds.): 15-34; New York: Academic Press.

WILSON, A. L., 1978 - Elemental analysis of pottery in the study of its provenance: A review. Journal of Archaeological Science, 5 (3): 219-236.

YOUNG, S. M. \& POLLARD, A. M., 2000 - Atomic spectroscopy and spectrometry. In: Modern analytical methods in art and archaeology (E. Ciliberto \& G. Spoto, eds.): 21-54; New York: Wiley.

YOUNG, W. J. \& WHITMORE, F. E., 1957 - Analysis of oriental ceramic wares by nondestructive x-ray methods. Far East Ceramic Bulletin, 9: I-27. 